\title{
Transient stimulation does not aid visual search: Implications for the role of saccades
}

\author{
EILEEN KOWLER \\ New York University, New York, New York 10003 \\ and \\ GEORGE SPERLING \\ New York University, New York, New York 10003 \\ and Bell Laboratories, Murray Hill, New Jersey 07974
}

\begin{abstract}
Previous demonstrations that performance on visual search tasks with briefly flashed presentations declines over time after the initial onset imply that saccades might be necessary for efficient acquisition of visual information. We imposed an additional abrupt onset and a displacement on character arrays containing letters and one numeral while subjects searched for the numeral. Presentations were always followed by a visual noise field. Presentation time varied $(50-800 \mathrm{msec})$. We found that performance with presentations containing one onset and remaining continuously visible was better than performance with presentations containing two onsets and containing displacements. Furthermore, information acquired near the onset of a continuous presentation was as effective as information acquired later. Our results demonstrate that abrupt onsets and displacements do not improve information acquisition with displays of alphanumeric characters. This finding is consistent with earlier reports that saccade-like retinal image motion does not contribute either to the maintenance of target visibility or to visual acuity.
\end{abstract}

Saccadic eye movements are obviously necessary for efficient visual information processing because they bring retinal images to the fovea where visual acuity is best. But this may not be the only useful role for saccades. Saccades can also aid visual information processing when visual details are already foveal and clearly distinct.

For example, Kowler and Steinman (1977) found that saccades were helpful for counting items that were haphazardly arranged in a 2-deg-diam display. In their experiment, subjects were required to count the items while either using saccades to scan around the display or not using saccades and maintaining the line of sight exclusively with slow eye movements at the center of the display. Counting accuracy was found to be $50 \%$ higher when saccades were used. But the reason that saccades were so helpful for counting was not that directing the line of sight at, or very near, items was necessary in order to see them distinctly. On the contrary, subjects noted that all the items could be easily distinguished when the

This research was supported by NIH Postdoctoral Fellowship SF32EY05235 to E. Kowler. We thank B. Fischer for serving as a subject, P. Burt, J. C. Falmagne, G. Iverson, M. Pavel, and R. M. Steinman for their useful suggestions, and I. Nicholson for typing the manuscript. Requests for reprints should be addressed to E. Kowler, Department of Psychology, 6 Washington Place, New York University, New York, New York 10003. line of sight was maintained at the center. This observation suggests that the difficulty subjects had in counting accurately when the line of sight was maintained at the center was not caused by an inability to resolve items at the peripheral portions of the display. This interpretation agrees with other reports that counting accuracy falls off before the limit of spatial resolution is reached (Atkinson, Campbell, \& Francis, 1976). Thus, some beneficial effect of saccades, other than improving visual acuity by bringing retinal images to the center of the fovea, will have to be found to explain why saccades improved counting accuracy.

Studies of visual search with briefly presented displays suggested that a possible reason saccades were helpful for counting was that transient stimulationin particular, an abrupt onset-may be essential for efficient visual information processing. The evidence for the belief that abrupt onsets are important comes primarily from an experiment by Sperling, Budiansky, Spivak, and Johnson (1971). They found that when subjects searched an array of characters for the presence of a single numeral, performance improved as time available to process the display increased, but most of the improvement occurred during the first $120 \mathrm{msec}$. Further increases in processing time had only negligible effects on performance. They interpreted their result as suggesting that the abrupt onset of a display initiates a brief period of information 
acquisition. If their suggestion is correct, then saccades should be very useful for efficient processing of visual information, because saccades create abrupt onsets by rapidly displacing the retinal image.

But Sperling et al.'s (1971) suggestion that onsets are necessary for efficient visual information processing could only be speculative because they had used displays that were turned off immediately after the onset. They presented a sequence of brief flashes of displays in which flashes were separated by dark intervals of various durations. Their finding that search became less efficient as the length of the dark interval increased means that the utility of the information being acquired from each single brief flash decreased with increasing time in the dark. Had displays remained visible, on the other hand, it seems likely that new information would continue to have been acquired long after the onset and performance would continue to have improved over time, rather than asymptote. The possibility that leaving displays visible would have improved performance is unlikely, however. Sperling and Melchner (1978) reported that performance in the same search task was as good for displays that were flashed briefly and followed by $240 \mathrm{msec}$ of darkness as it was for displays that remained continuously visible during the first $200 \mathrm{msec}$. Their result shows that the continued presence of a display long after the onset was not able to provide new information. This result strengthens the suggestion that abrupt onsets initiate a brief period of information acquisition because both the briefly flashed displays and the continuously visible displays contained one abrupt onset, and both produced the same performance. ${ }^{1}$

The experiments reported here were intended to provide further support for the importance of onsets in visual information processing. Using the same visual search task employed by Sperling et al. (1971) and by Sperling and Melchner (1978), we sought to answer three questions:

(1) Would Sperling and Melchner's (1978) finding that performance is the same with displays that contain one onset (briefly flashed and continuously visible displays) be obtained at many different presentation times? To find out, we compared performance with displays that were briefly flashed and followed by a variable interval of darkness (50 to $800 \mathrm{msec}$ ) to performance with displays that remained visible during the entire interval. To terminate the availability of the displays for processing, a visual noise field always was presented after the dark interval following the briefly flashed displays and immediately after the continuous presentations.

(2) Is performance improved by a second onset? To find out, performance with displays that contained two onsets was compared to performance with the displays that contained only one onset. Two onsets were provided by presenting two brief flashes of the display, one at the beginning and one at the midpoint of the dark interval.

(3) Must a second onset appear at a different retinal location from the first in order to be effective? To find out, performance with the two-flash presentations in which both flashes appeared in the same location was compared to performance with the two-flash presentations when the second flash was displaced relative to the first. Also, performance with continuous presentations was compared to performance with continuous presentations in which a single rapid displacement occurred at the midpoint of the presentation. Determining the effect of displacements in continuous presentations was particularly relevant to the question of the role of saccades in visual information processing because this stimulus, of all those we tested, most closely resembles what occurs naturally when saccades rapidly displace the retinal image.

\section{METHOD}

\section{Stimuli}

Stimuli were similar to those used by Sperling et al. (1971) and Sperling and Melchner (1978). Stimuli were arrays of alphanumeric characters that were displayed on a CRT screen (DEC VT04 display-console with a fast-decay P-4 phosphor, controlled by a PDP-15 computer). The screen was located $1.5 \mathrm{~m}$ from the subject. Stimuli were viewed binocularly, and the subject's head was stabilized by a biteboard. A biteboard was used both to preserve a fixed distance between the subject's head and the screen and to prevent high-velocity $(.5$ to $5 \mathrm{deg} / \mathrm{sec})$ retinal image motions that result from the slow oculomotor subsystems' failure to fully compensate for head rotations (Skavenski, Hansen, Steinman, \& Winterson, 1979; Steinman \& Collewijn, in press). Retinal image velocities, when the head is supported by a biteboard, are much lower (less than $.25 \mathrm{deg} / \mathrm{sec}$; Skavenski et al., 1979).

Two kinds of stimulus displays were generated: character arrays and noise fields. The character array was the stimulus for the search task. It was composed of 23 letters and one numeral arranged in a 5 by 5 square matrix subtending $4.3 \mathrm{deg}$ on a side. Any of the numerals 0 through 9 could appear. Any letter could appear except $B, I, O, Q$, and $Z$, which were excluded because of their close resemblance to the numerals $3,1,0$, and 2 . The center location of the array always contained the fixation target: two dots separated vertically by $11 \mathrm{~min}$ arc.

The visual angle subtended by the individual characters was $16 \mathrm{~min}$ arc horizontally and $23 \mathrm{~min}$ arc vertically. Their centerto-center separations were $1.0 \mathrm{deg}$ horizontally and $.6 \mathrm{deg}$ vertically. All characters could be easily identified when the center of the array was fixated.

The second kind of display generated was a visual noise field which served the purpose of erasing any visual persistence of the character arrays (Sperling, 1963). The noise field was a 15 by 15 array of closely spaced letters. The noise field subtended $6.5 \mathrm{deg}$ on a side.

The characters were illuminated briefly $(<1 \mathrm{msec})$ to a luminous directional energy (LDE) of .16 cd- $\mu \mathrm{sec} / \mathrm{mm}$ (see Sperling, 1971). The mean LDE per flash for a character was $4.6 \mathrm{~cd}-\mu \mathrm{sec} /$ character (range 2.6 to $7.1 \mathrm{~cd}-\mu \mathrm{sec} / \mathrm{character}$ ).

The characters appeared medium bright when viewed against a $\operatorname{dim}(.4 \mathrm{ml})$ background that was provided by two incandescent lamps that illuminated the screen. Otherwise, the room was dark. 


\section{Procedure}

Pretrial. Before each trial, the subjects fixated the two-dot fixation target. When they were ready, they pressed a button which caused the character array to appear $250 \mathrm{msec}$ later.

Types of presentations during trials. Character arrays were presented in one of the following five different ways on any trial (sec Figure 1): (1) Single flash: A single 1-msec flash of a character array followed by a dark interval of duration T msec. (2) Two flashes: Two 1-msec flashes of the same character array, one at the beginning and one at the midpoint of a dark interval of duration I msec. (3) Continuous: A single character array continuously presented for duration T msec. (4) Two flashes displaced: Two 1-msec flashes of the same character array, one at the beginning and one at the midpoint of the dark interval of duration $\mathrm{T}$ msec, with the second flash displaced one character width (16 min arc) to the right or to the left of the first. This size displacement was chosen for two reasons. First, with this size displacement, no character of the second flash appeared in a location that was previously occupied by a character of the first flash. Second, the displacement was small enough so that all the characters remained close enough to the center of the visual field to be easily identified after the displacement. (5) Continuousdisplaced: Two consecutive continuous presentations of the same character array, each of duration $\mathrm{T} / 2 \mathrm{msec}$, with the second presentation displaced one character width $(16 \mathrm{~min}$ arc) to the right or to the left of the first.

The continuous presentations were actually composed of a series of brief flashes of the character array repeated at $25-\mathrm{msec}$ intervals $(40 \mathrm{~Hz})$. This refresh interval was short enough so that individual flashes could not be seen and the display appeared to be continuous. The fixation dots also were presented in this manner. The fixation dots remained on during dark intervals and remained stationary even on trials in which displacements occurred as a guide for maintaining a steady line of sight.

The visual noise field was presented for $2 \mathrm{sec}$ after the last dark interval of the briefly flashed presentations, and following the termination of the continuous presentations. Presentation time is defined as the time from the first appearance of the character array to the appearance of the noise field. Presentation time on any trial was chosen to be $50,100,200,400$, or $800 \mathrm{msec}$.

Eye movements. Subjects were instructed to suppress saccades and to maintain a steady line of sight on the fixation dots during the time that displays were presented. The longest presentation time $(800 \mathrm{msec})$ was chosen to be short enough so that the occurrence of any spontaneous saccades would be rare. ${ }^{2}$

Reports. After the character array and noise field were presented, the subjects reported, by pressing appropriate, numbered buttons, the numeral they had seen, its location in the matrix (row and column), and a confidence rating on a 3-point scale for both the identification and the location reports. Subjects were required to report all of these after every trial, guessing identity and location if necessary. After each report, they were shown the character array so that they would know the correct answer.

\section{Design}

A mixed list design was used. The identity and location of the target numeral and of the letters, the type of presentation, the presentation time, and the direction of a displacement (when applicable) were all chosen randomly, independently, and with

Figure 1. Sequence of display frames for the five different types of presentations illustrated for the $200-$ msec presentation time. Each rectangle denotes a brief presentation of either the character array (small rectangle) or the visual noise field (large rectangle). Labels indicate the different types of presentations: single flash (SF), two flashes (2F), continuous (C), two flashes displaced (2F-D), and continuous-displaced (C-D). The interflash interval for the continuous presentations and the noise field is $\mathbf{2 5}$ msec. Only the first four flashes of the noise field are shown; during the experiment, it was presented for 2 sec. replacement. The subject never knew what these choices were before a trial.

\section{Subjects}

Two subjects were tested. One (E.K.) was one of the authors. She had previous experience as a subject in both psychophysical
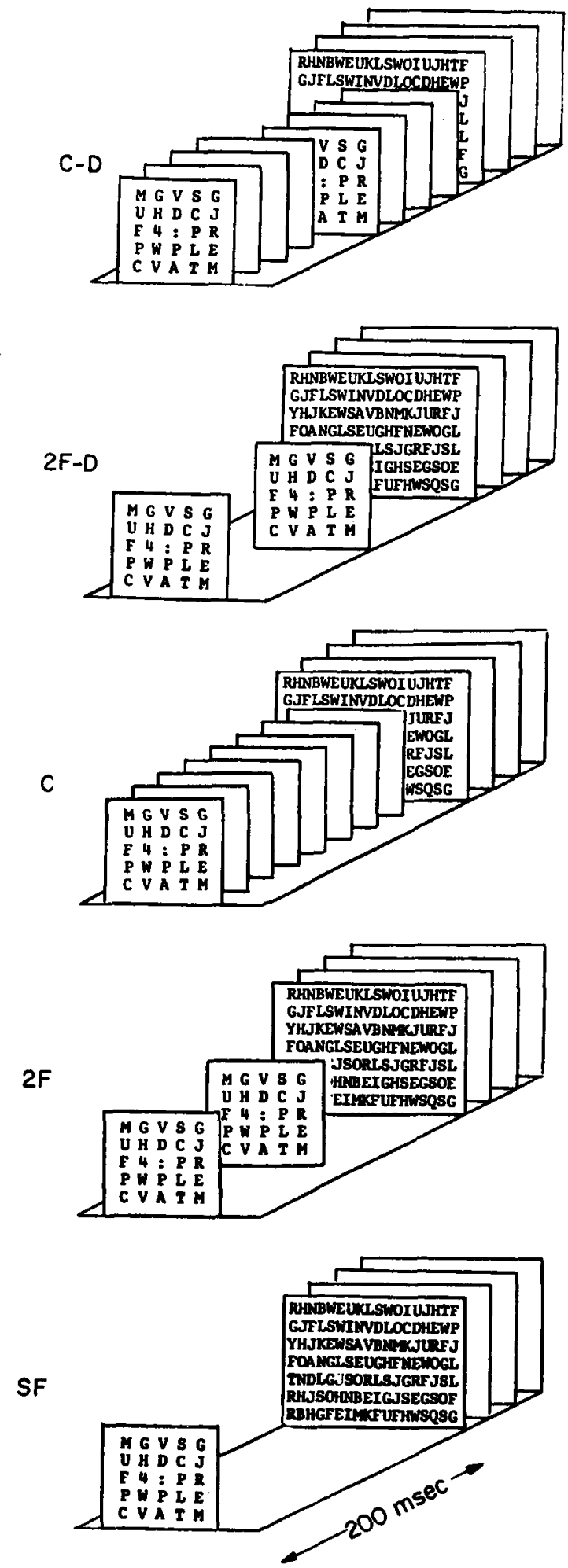
and oculomotor experiments. The other (B.F.) was naive as to the purpose of the experiment and had no previous experience as a subject. Both subjects practiced the search task until performance stabilized before the data reported here were collected.

\section{RESULTS}

\section{Information Acquisition Does Not Depend on the Number of Onsets}

If onsets are required to initiate information acquisition, as we have suggested, then we would expect to find: (1) the same performance with the single-flash and the continuous presentations because each contained only one onset, and (2) better performance with the two-flash presentations than with both the single-flash and the continuous presentations because the two-flash presentations contained two onsets. If a second onset were to provide useful information only when presented at a new retinal location, then we would expect to find (3) the best performance with the two-flash-displaced and continuous-displaced presentations.

None of these expectations was met: (1) Performance with continuous presentations was always better than performance with the single-flash presentations for times longer than $100 \mathrm{msec}$. Thus, contrary to the Sperling and Melchner (1978) result, the presence of the display long after the initial abrupt onset was useful for visual search. (2) Adding a second onset helped only in that performance with two flashes was better than performance with a single flash. But two flashes, containing two onsets, never produced better performance than continuous presentations containing one onset. (3) The expected benefits of a second onset did not emerge when the second onset was displaced relative to the first. Displacements did not improve performance for either the two-flash or the continuous presentations. These results are summarized in Figure 2, which shows the proportion of trials in which the numeral was both identified correctly and located correctly as a function of time for each of the five types of presentations. Similar results were also obtained for other response measures, namely, the probability of identifying the numeral correctly regardless of the report of location, and the conditional probability of locating the numeral correctly given that a correct identification had occurred.

A further analysis of the effects of displacements is desirable because the presentations with displacements, particularly the continuous-displaced presentations, came closest to mimicking the effects of saccades on the retinal image. Displacements were helpful in only two of the possible 20 cases (at $800 \mathrm{msec}$ with continuous presentations for E.K. and at $200 \mathrm{msec}$ with two-flash presentations for B.F.), but in neither case was the improvement statis-

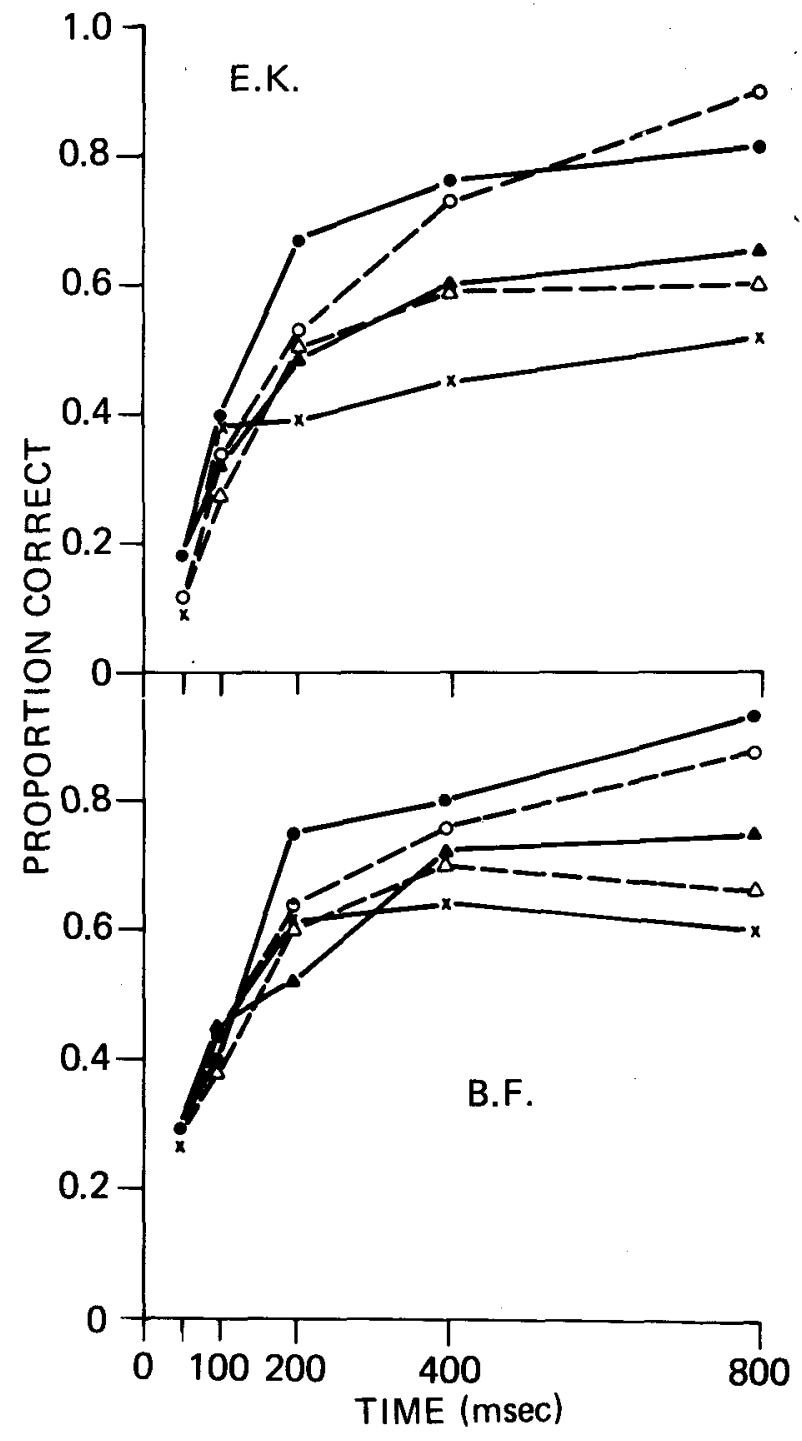

Figure 2. Proportion of trials in which the target numeral was both identified correctly and located correctly as a function of presentation time for subjects E.K. and B.F. for the five different types of presentation: single flash (crosses), two flashes (filled triangles), continuous (filled circles), two flashes displaced (open triangles), and continuous-displaced (open circles). Each datum point is based on approximately 100 observations.

tically significant. In fact, displacements with continuous presentations tended to impair performance. E.K.'s performance, pooled over all presentation times, was significantly worse with the continuousdisplaced presentations than with continuous presentations (proportion correct with continuous presentations $=.58, \mathrm{~N}=512$; with continuous-displaced $=.49, \mathrm{~N}=506 ; \chi^{2}=6.9, \mathrm{df}=1, \mathrm{p}<.01$ ). Performance with continous presentations was impaired by displacements for B.F. as well, but in her case the difference was not significant (proportion correct with continuous $=.63, \mathrm{~N}=501$; with continous- 
displaced $=.58, \mathrm{~N} \doteq 478, \chi^{2}=2.1, \mathrm{df}=1, \mathrm{p}>$ .10). Displacements with two-flash presentations did not produce any effects on performance, good or bad, for either subject.

In short, these results argue against the importance of abrupt onsets and displacements in visual information processing. Onsets can be useful insofar as a second flash may refresh a fading memory trace left behind by the first flash. But, contrary to Sperling et al.'s (1971) suggestion and Sperling and Melchner's (1978) result, additional onsets are not required for this refreshment. Our results suggest that the continued presence of a display can provide new information because we found that performance was best when displays were turned on once, and then remained on and stationary.

Comparison with Sperling and Melchner's (1978) result. Our results differed from Sperling and Melchner's (1978) results. We did not find, as they did, that performance with single, briefly flashed displays was the same as performance with continuous displays at a presentation time of $200 \mathrm{msec}$. Note, however, that we did find that performance with the single-flash presentations was about the same as performance with continuous presentations at shorter (50- and 100-msec) times. So it is possible that Sperling and Melchner's (1978) subject was able to use the visual persistence of the single brief flash longer than our subjects could, either because of subject differences or because of stimulus differences. The speculation that subject or stimulus differences made visual search with single brief flashes more efficient in Sperling and Melchner's experiment than in our experiment is plausible because their subject searched as accurately as our subjects did, but with a much larger display than we used. A systematic investigation of the reasons for the differences between our results and Sperling and Melchner's might be enlightening, but would probably not lead to new insights about the role of onsets for the following reason. Finding that performance with a single brief flash can be the same as performance with a continuous presentation at short presentation times may simply imply that under certain conditions the persistence of a single brief flash may be sufficient to provide useful information for at least $100 \mathrm{msec}$, or sometimes $200 \mathrm{msec}$. It does not imply that abrupt onsets are necessary for efficient processing. On the contrary, our finding that performance with continuous presentations containing one onset, is better than performance with presentations containing two onsets and presentations containing displacements suggests that additional abrupt onsets and imposed displacements are not necessary.

\section{Information Acquisition Continues Long After the Initial Abrupt Onset}

The superiority of performance with continuous presentations over performance with single-flash presentations means that information was still being obtained from a continuous presentation long after its onset. But the observed rate of improvement of performance over time with continuous presentations may still have implications for the role of onsets. Note that the most rapid improvment in performance with continuous presentations occurred during the first $200 \mathrm{msec}$ (see Figure 2). After that, the rate of improvement slowed down. This may mean that, although new information was acquired from the continuous presentations after the onset, more information was acquired during the initial portion of the presentation than during the latter portion. This outcome would argue for the importance of onsets.

However, a decrease in the rate of improvment of performance does not necessarily mean that information acquisition slows down over time. Information acquisition may continue unchanged over time, but redundant information may be acquired. If redundant information were acquired, then a decrease in the rate of improvement in performance would be expected, because redundant information would not be as useful for finding the target numeral as would new information. This interpretation would argue against onsets having any special role in initiating information acquisition. We will now show that a model which incorporates the acquisition of redundant information can adequately describe the rate of improvement in performance over time that we have observed.

Independent sampling model. An example of a process which would lead to the accumulation of redundant information is as follows. Suppose the subject continually samples information from displays during the entire presentation. A sample might consist, for example, of some or all of the features contained in a subset of the 24 locations of the character array. If enough features from the location containing the target numeral are included in any sample, then the numeral is discovered. Furthermore, suppose that the sampling process has two additional characteristics. First, all the samples taken in the first half of a continuous presentation (or in the first flash of a two-flash presentation) are as useful (i.e., contain the same number of features or locations) as all the samples taken in the second half (or, in the second flash). Second, samples are taken independently. That is, the particular features or locations that are acquired in the $\mathrm{n}^{\text {th }}$ sample does not determine the particular features or locations that are acquired in the $(n+1)^{\text {th }}$ sample. It is this independence that produces redundancies, because each sample after the first may include features or locations that have already been acquired in previous samples [See Loftus (1972), Loftus \& Kallman (1979), and Rumelhart (1970) for descriptions of similar models.]

The way we formulated and tested the ability of 
this kind of independent sampling model to predict our data can be illustrated by considering what would happen in a continuous presentation lasting $2 \mathrm{~T} \mathrm{msec}$ if the model holds. We assume that the utility of samples is the same in the first and in the second $T$ msec. Let the probability that the numeral is found in either half of the $2 \mathrm{~T}$ msec presentation be $\mathrm{p}$. The probability that the numeral is not found in either half is $(1-p)$. For a presentation lasting $2 \mathrm{~T} \mathrm{msec}$, consisting of the two independent halves, the probability that the numeral is not found is the product of the probabilities that it is not found in either half, or $(1-p)^{2}$. Thus, the probability that the numeral is found in $2 \mathrm{~T} \mathrm{msec}\left(\mathrm{P}_{2}\right)$ is $1-(1-p)^{2}$. Simplifying the expression leads to:

$$
\mathbf{P}_{2}=p+p(1-p)
$$

The probability $\left(P_{1}\right)$ that the numeral is found in either half, that is, in any presentation lasting $\mathrm{T} \mathrm{msec}$, is simply $\mathrm{p}$.

To test the model, we compared actual performance for presentation times, $T$ and $2 \mathrm{~T}$ msec, with predicted performance, $P_{1}$ and $P_{2}$. A prediction of $P_{1}$ and $P_{2}$, as Equation 1 shows, requires an estimate of p. This estimate was obtained by finding the value of $p$ that simultaneously minimized the chi square of the difference between observed and predicted performance for presentation times of both $T$ and $2 \mathrm{~T} \mathrm{msec.}{ }^{3}$

A further refinement in estimating $p$ is needed. For any pair of presentation times, $T$ and $2 \mathrm{~T}$ msec, a different $p$ was estimated for trials in which easy and difficult target numerals were presented. Target difficulty depended mainly on the identity of the numeral (e.g., $7 \mathrm{~s}$ were easier to find than $5 \mathrm{~s}$ ) and on the location (e.g., numerals in the central locations were easier to find than numerals in more peripheral locations). Separate analysis of trials with targets of different difficulties was necessary because a prediction of performance at $2 \mathrm{~T} \mathrm{msec}$ would be too large if the target difficulties were too heterogeneous. ${ }^{4}$

Trials were grouped into four classes of difficulty by the following scheme. Performance with each target numeral and at each location (for all types of presentations and all presentation times) was examined to determine which targets were easiest and which were the most difficult. Numerals were divided into two classes (the five easiest and the five hardest) and locations were divided into two classes (the 12 easiest and 12 hardest). Combining these two pairs of classes produced four classes of targets. This scheme was successful in reducing the heterogeneity of target difficulties. The proportions of correct reports in each class for E.K. were $.77(\mathrm{~N}=612)$, $.57(\mathrm{~N}=615), .36(\mathrm{~N}=566)$, and $.23(\mathrm{~N}=591)$. The proportions for B.F. were $.84(\mathrm{~N}=557), .62$ $(\mathrm{N}=614), .54(\mathrm{~N}=578)$, and $.31(\mathrm{~N}=534)$.
After trials for each pair of presentations times ( $T$ and $2 \mathrm{~T} \mathrm{msec}$ ) were separated into the four classes, a different value of $p$ was estimated for each class by minimizing chi squares as described above. The minimunı chi squares were then summed over the four classes to give one chi square for each pair of presentation times. Similarly, the predicted outcomes, $P_{1}$ and $P_{2}$, for each pair of presentation times were determined by combining the predictions for each of the four classes.

The independent sampling model is a good predictor of performance. The predicted performance for con-

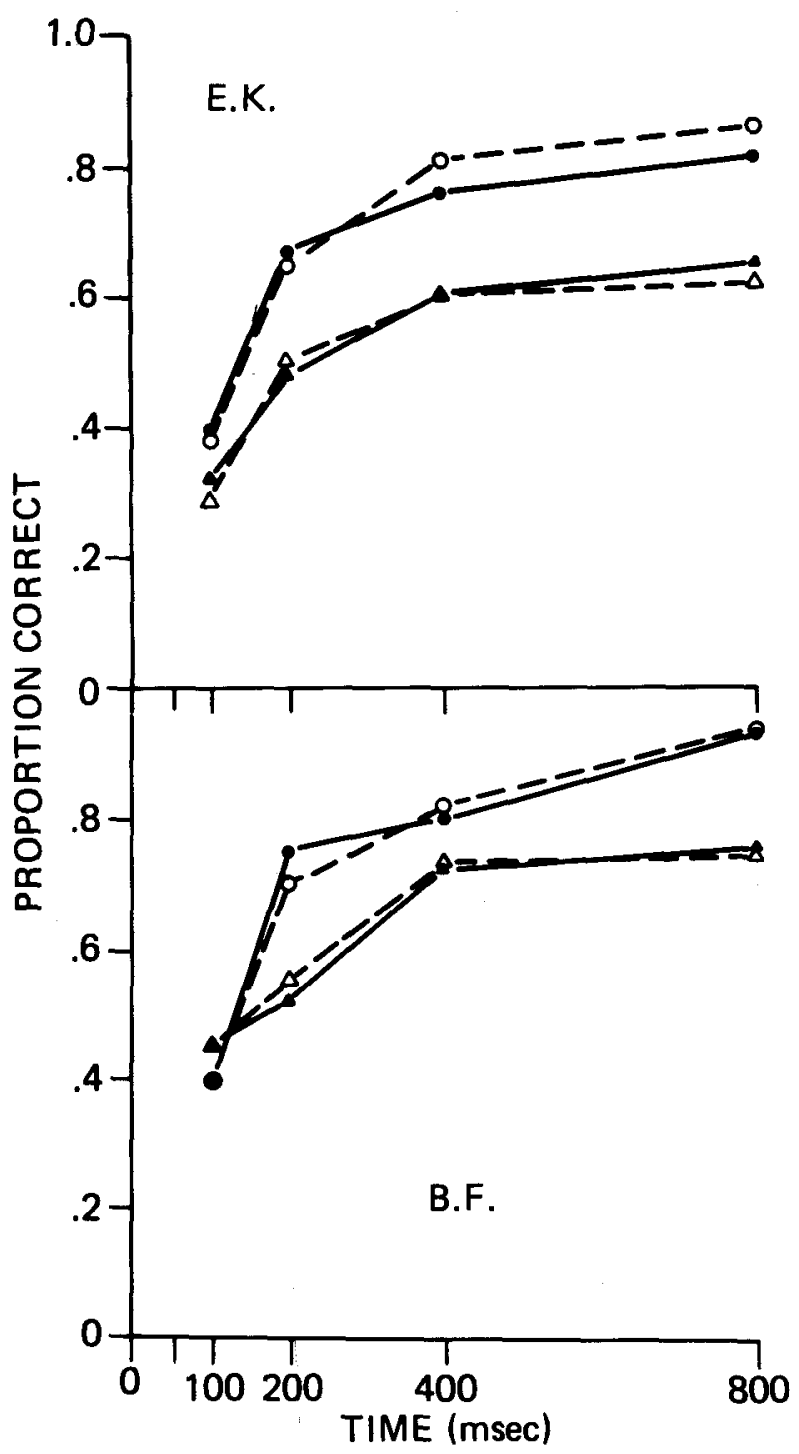

Figure 3. Predicted performance $\left(P_{2}\right)$ based on independent sampling for continuous (circles) and two-flash (triangles) presentations lasting 2T msec (see text). Observed (filled symbols) and predicted (open symbols) proportions of trials in which the target numeral was both identified correctly and located correctly are shown as a function of presentation time for subjects E.K. and B.F. Each datum point is based on approximately 100 observations. 
Table 1

Test of Independent Sampling Model

\begin{tabular}{|c|c|c|c|c|c|c|c|c|c|c|c|c|c|c|c|c|}
\hline \multirow[b]{3}{*}{ Presentation } & \multicolumn{16}{|c|}{$2 \mathrm{~T} \mathrm{msec}$} \\
\hline & \multicolumn{4}{|c|}{100} & \multicolumn{4}{|c|}{200} & \multicolumn{4}{|c|}{400} & \multicolumn{4}{|c|}{800} \\
\hline & $P_{1}$ & $P_{2}$ & $\mathrm{df}$ & $x^{2}$ & $P_{1}$ & $\mathbf{P}_{2}$ & df & $x^{2}$ & $P_{1}$ & $\mathrm{P}_{2}$ & $\mathrm{df}$ & $x^{2}$ & $P_{1}$ & $P_{2}$ & $\mathrm{df}$ & $x^{2}$ \\
\hline & \multicolumn{16}{|c|}{ Subject E.K. } \\
\hline$C \rightarrow C$ & .21 & .38 & 4 & 2.49 & .43 & .65 & 4 & 2.35 & .59 & .81 & 4 & 6.41 & .70 & .86 & 3 & 7.22 \\
\hline$S F \rightarrow 2 F$ & .17 & .28 & 2 & $6.71 *$ & .34 & .50 & 4 & 6.02 & .39 & .60 & 4 & 2.99 & .49 & .62 & 4 & $10.59 *$ \\
\hline$C \rightarrow C-D$ & .19 & .34 & 4 & 2.10 & .37 & .55 & 4 & 1.32 & .59 & .79 & 4 & 7.37 & .74 & .91 & 3 & 5.19 \\
\hline \multirow[t]{2}{*}{$S F \rightarrow 2 F-D$} & .15 & .22 & 3 & 6.60 & .32 & .53 & 4 & 5.86 & .38 & .59 & 4 & 3.01 & .46 & .59 & 3 & 1.76 \\
\hline & \multicolumn{16}{|c|}{ Subject B.F. } \\
\hline $\mathrm{C} \rightarrow \mathrm{C}$ & .28 & .40 & 4 & 3.17 & .46 & .70 & 3 & 3.95 & .72 & .82 & 3 & 6.44 & .77 & .94 & 2 & 1.42 \\
\hline$S F \rightarrow 2 F$ & .26 & .45 & 4 & 1.30 & .41 & .55 & 4 & 4.51 & .57 & .73 & 3 & 1.43 & .60 & .74 & 3 & 4.22 \\
\hline$C \rightarrow C-D$ & .28 & .45 & 3 & $10.00^{*}$ & .37 & .66 & 4 & 2.08 & .62 & .81 & 3 & $9.40^{*}$ & .75 & .91 & 2 & 2.24 \\
\hline$S F \rightarrow 2 F-D$ & .23 & .41 & 3 & 2.51 & .40 & .61 & 4 & 6.61 & .51 & .70 & 4 & $12.10^{*}$ & .54 & .70 & 3 & $12.66 \dagger$ \\
\hline
\end{tabular}

Note-Entries indicate the predicted performance at $T$ msec $\left(P_{1}\right)$, at $2 T$ msec $\left(P_{2}\right)$, and the chi square for each pair of predictions tabled under the $2 T$-msec presentation time. Presentation indicates the type of presentation used at $T$ msec (left of arrow) and the type of presentation used at $2 T$ msec (right of arrow) to generate each $P_{1}$ and $P_{2} . S F=$ single $\cdot$ flash, $C=c o n t i n u o u s, 2 F=t w o-f l a s h$, $C-D=$ continuous-displaced, and $2 F-D=$ two-flash-displaced presentations. Each prediction $\left(P_{1}\right.$ and $\left.P_{2}\right)$ is the weighted mean of the predictions of the four stimulus classes. Each value of the chi square is the sum of the individual chi squares obtained for each of the four stimulus classes (see text). Degrees of freedom differ because stimulus classes that contained cells with less than one predicted observation were omitted from the chi-square analysis. ${ }^{*} p<.05 . \quad t_{p}<.01$.

tinuous presentations was reasonably close to the observed performance. The chi-square values, shown in Table 1 along with the predictions $P_{1}$ (or, equivalently $p$, since $P_{1}=p$ ) and $P_{2}$, indicated that none of the departures of the data from the predicted performance was significant. A graphic description of the closeness of fit is shown in Figure 3, which compares predicted performance, $\mathbf{P}_{2}$, with observed performance for each of the $2 \mathrm{~T}-\mathrm{msec}$ presentation times.

The same analysis was also applied to the twoflash presentations. Predictions were based on the observed performance at $\mathrm{T}$ msec with single-flash presentations and the observed performance at $2 \mathrm{~T} \mathrm{msec}$ with the two-flash presentations. In most cases, the independent sampling model provided a good description of performance. There were two significant departures, both for E.K. at 100 and $800 \mathrm{msec}$ (see Table 1). In these two cases she performed slightly better with the two-flash presentation than predicted by the independent sampling model (see Figure 3 ). These two departures, however, do not present a convincing case for the importance of onsets, because neither E.K.'s performance at 200 and $400 \mathrm{msec}$ nor B.F.'s performance at all four of the presentation times differed significantly from the model's predictions.

Predictions of the independent sampling model for performance when displacements occurred is shown in Figure 4 and Table 1. Predictions for the twoflashed-displaced presentations were based on the observed performance at $\mathrm{T}$ msec with single-flash presentations, and the observed performance at 2T msec with two-flash displaced presentations. Pre- dictions for the continuous-displaced presentations were based on the observed performance at $T$ msec with continuous presentations, and the observed performance at $2 \mathrm{~T}$ msec with continuous-displaced presentations. There were four significant departures, all for B.F. In each of these cases, displacements had detrimental effects, that is, she did not perform as well as the model predicted. E.K.'s performance, however, was accurately described by independent sampling. ${ }^{\mathrm{s}}$

To summarize, the independent sampling model accurately predicted performance with continuous presentations and, in most cases, with other types of presentations. ${ }^{6}$ Thus, the model confirms our earlier suggestion that extra onsets are not required for efficient information acquisition. The second onset in a two-flash presentation does provide enough information for a second set of independent samples, but so does the second half of a continuous presentation. That fact that the two-flash presentation contains two onsets and the continuous presentation contains only one onset makes little difference. Information acquisition continues throughout a presentation, and additional onsets and displacements are not required to revive information acquisition.

Alternatives to the independent sampling model. Recall that the test of the independent sampling model incorporated the assumptions that samples contain the same number of features or locations no matter when they occur during a presentation, and that samples are taken independently. These assumptions may, of course, be false. Consider the following alternative models. (1) Successive samples are taken from different portions of the array and contain 


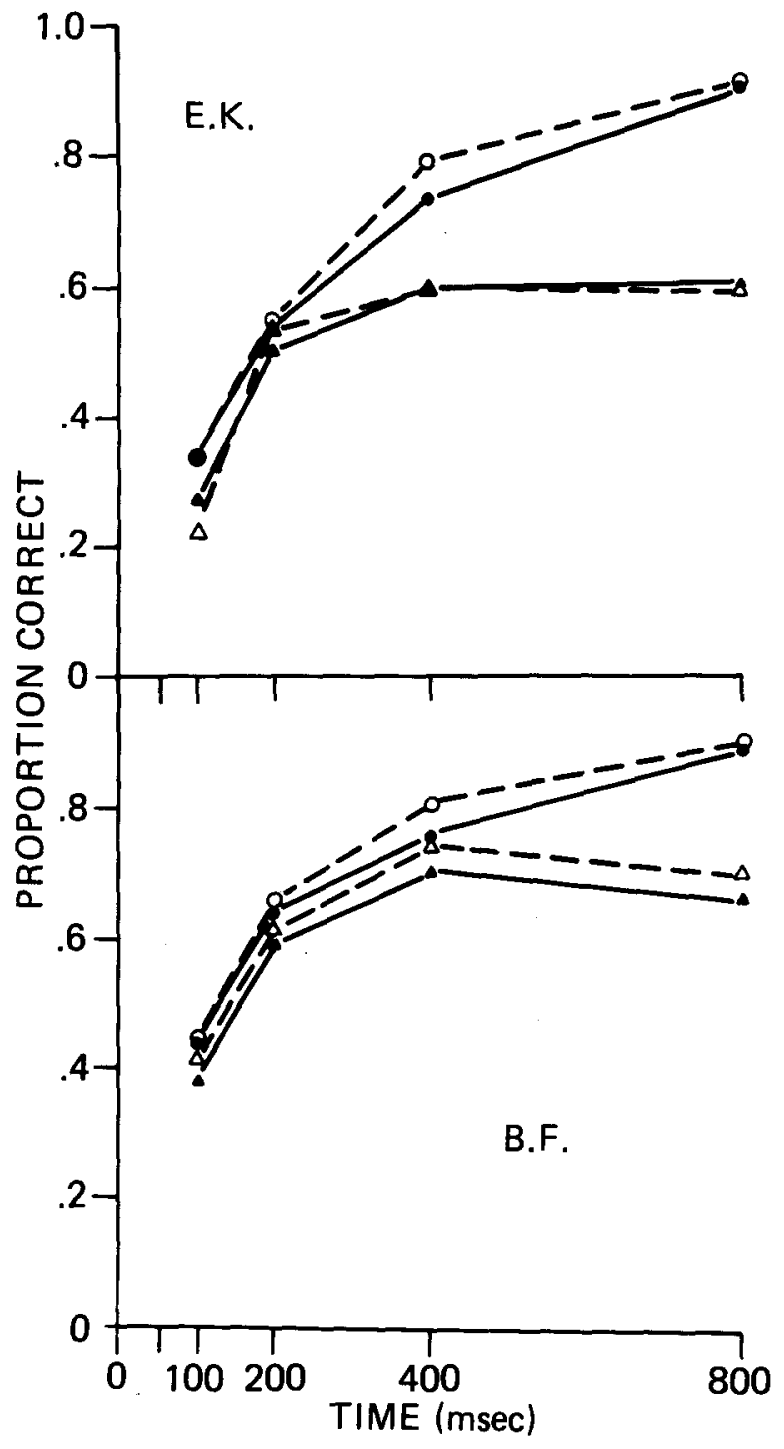

Figure 4. Predicted performance $\left(P_{2}\right)$ based on independent sampling for continuous-displaced (circles) and two-flash-displaced (triangles) presentations lasting $2 \mathrm{~T}$ msec (see text). Observed (filled symbols) and predicted (open symbols) proportions of trials in which the target numeral was both identified correctly and located correctly are shown as a function of presentation time for subjects E.K. and B.F. Each datum point is based on approximately 100 observations.

fewer features or locations as presentation time increases. (2) Successive samples are taken from the same portion of the array and contain more features or locations as presentation time increases. Each of these alternative models could provide an equivalent description of our data, so we cannot distinguish among all of these processes. But note, these processes are all alike in that they suggest that the earlier portions of a presentation are as useful as the latter portions, that is, the deleterious effects of decreasing the number of features or locations in a sample over time are canceled by benefits derived from systematically sampling from different portions of the array. Thus, both the independent sampling model and its equivalents have the same implications for the evaluation of the role of onsets: They describe ways in which overall performance can improve at a slower rate over time without requiring that information acquired near onsets be more useful than information acquired later in a presentation.

We can therefore conclude that neither the observed rate of increase in performance over time nor the observed relationship between performance with the different types of presentations provides evidence that onsets are necessary for efficient information acquisition.

\section{DISCUSSION}

Our results do not support the notion that information acquisition is initiated by abrupt onsets. We found that performance on a visual search task was best when displays contained only one onset and remained continuously visible and stationary. Neither an additional onset (i.e., two brief flashes) nor an imposed displacement produced better performance than the continuous, stationary displays. Furthermore, the fit of the independent sampling model suggests that acquisition of information with such continuous presentations proceeds undisturbed over time. Thus, new onsets are not required to allow efficient continued use of the information in the display. These findings make it unlikely that the contribution of saccades to visual information processing lies in benefits derived from their creation of abrupt onsets. Indeed, the stimulus that most closely mimicked the effect of saccades on the retinal image-the continuous-displaced presentation-tended to have slightly detrimental effects on performance.

\section{Comparison with Prior Studies \\ of the Effects of Onsets}

Ours is not the only report which suggests that onsets may not be important for visual information processing. Loftus (Note 1) recently showed that recognition memory for pictures improves with increasing exposure duration, and that adding onsets by dividing an exposure into short presentations (50 to $200 \mathrm{msec}$ ) separated by masks has detrimental effects on performance.

Studies of visual acuity also confirm the suggestion that onsets are not important. Baron and Westheimer (1973) used a Landolt-C target and reported that acuity improved as exposure duration increased up to $800 \mathrm{msec}$ (the longest duration they tested). They also measured acuity when onsets were added by presenting two $100-\mathrm{msec}$ exposures of the target, separated by various intervals of darkness. They found that acuity tended to improve with increasing flash separation, just as it had improved with increas- 
ing exposure duration. They concluded that: "Rather than being affected by the extra onsets and cessations, the system acts as though it were handling the asynchronous presentations in the same manner as the uniform presentations"' (p. 219).

Thus, our findings on the role of onsets in visual search are similar to Baron and Westheimer's (1973) findings on the role of onsets in visual acuity. Had it turned out that additional onsets had beneficial effects in either of the two tasks, it would have suggested that the visual system responds differently to the temporal pattern of stimulation when subjects are faced with an acuity task than when they are required to do something that may be more complex, namely, search for a target. But as it stands, there is no evidence that these two different kinds of psychophysical tasks require different kinds of retinal stimulation, at least to the extent that additional onsets are not helpful for either. This suggests that the kind of retinal stimulation that optimizes performance of "complex" tasks, such as visual search and picture recognition, may be the same kind of retinal stimulation that optimizes the resolution of visual details. This suggestion is supported further by comparisons of our results with results from experiments on vision with stabilized images when various kinds of retinal image motions are imposed.

\section{Comparison with Studies of Vision with Stabilized Images}

These studies have shown that imposed saccadelike motions are not optimal for either preventing the disappearance of stabilized targets or improving their contrast sensitivity. Gerrits and Vendrik (1974), for example, reported that fading of stabilized targets can be prevented more effectively by imposed stimulus motions that resemble the irregular low-velocity oscillations of the eye (they used bandwidth-limited Gaussian noise) than by imposed stimulus motions that resemble saccades (binary noise). Similarly, King-Smith and Riggs (1978) found that contrast sensitivity of stabilized targets was greater with imposed constant velocity oscillations (triangular motion) than with imposed rapid jumps of the image (square-wave motion). Although they did not conclude that square-wave motion was useless, in the only case in which square-wave motion was found to produce greater contrast sensitivity than triangular motion, at amplitudes of $4 \mathrm{~min}$ arc or less, the differences were small and not statistically reliable.

We found that saccade-like image motions, that is, rapid displacements, do not improve visual search. The similarity between our results and these prior experiments on visibility and contrast sensitivity agrees with the suggestion made above that the kind of retinal image motion that facilitates information acquisition in a visual search task and the kind of retinal image motion needed to see and resolve visual detail may be the same. This kind of motion is likely to be the low-velocity oscillations characteristic of slow eye movements, and not the abrupt displacements produced by saccades.

\section{The Role of Saccades in \\ Visual Information Processing}

The problem that inspired our experiment still remains unsolved: Is there any role for saccades in visual information processing other than the obvious role of improving visual acuity by bringing targets to the fovea? Neither our results nor prior work supports the notion that saccades are useful because they produce new onsets. It is possible that there are beneficial effects of saccades, and these effects emerge only when the oculomotor response occurs. There are results supporting such a suggestion. Wolf, Huaske, and Lupp (1978) have evidence that there may be a postsaccadic enhancement of contrast sensitivity that occurs when saccades are made to a grating that had previously been seen in the periphery of the visual field. Confirmation of such beneficial effects of saccades requires a comparison of performance in a visual task when saccades are used to shift the line of sight with performance when retinal displacements of the same size, direction, and frequency are created by moving the visual display.

\section{REFERENCE NOTE}

1. Loftus, G. Tachistoscopic simulations of eye fixations on pictures. Manuscript submitted for publication.

\section{REFERENCES}

Atrinson, J., Campbell, F. W., \& Francis, M. R. The magic number $4 \pm 0$ : $A$ new look at visual numerosity judgments. Perception, 1976, 5, 327-334.

Baron, W. S., \& Westheimer, G. Visual acuity as a function of exposure duration. Journal of the Optical Society of America, 1973, 63, 212-219.

Ciuffreda, K. J., Kenyon, R. V., \& Stark, L. Suppression of fixational saccades in stalismic and anisometropic amblyopia. Oththalmic Research, 1979, 11, 31-39.

Gerrits, H. J. M., \& Vendrik, A. J. H. The influence of stimulus movements on perception in parafoveal stabilized vision. Vision Research, 1974, 14, 157-180.

Haber, R. N., \& Nathanson, L. S. Processing of sequentially presented letters. Perception \& Psychophysics, 1969, 5, 359-361.

Haddad, G. M., \& Steinman, R. M. The smallest voluntary saccade: Implications for fixation. Vision Research, 1973, 13, 1075-1086.

Haddad, G. M., \& Winterson, B. J. Effect of flicker on oculomotor performance. In G. Lennerstrand \& P. Bach-y-Rita (Eds.), Basic mechanisms of ocular motility and their clinical implications, Oxford: Pergamon, 1975.

King-Smith, P. E., \& Riggs, L. A. Visual sensitivity to controlled motion of a line or edge. Vision Research, 1978, 18, 1509-1520.

Kowler, E., \& Steinman, R. M. The role of small saccades in counting. Vision Research, 1977, 17, 141-146. 
Kowler, E., \& Steinman, R. M. Miniature saccades: Eye movements that do not count. Vision Research, 1979, 19, 105-108.

Kowler, E., \& Steinman, R. M. Small saccades serve no useful purpose. Vision Research, in press.

LofTus, G. Eye fixations and recognition memory for pictures. Cognitive Psychology, 1972, 3, 525-551.

Loftus, G., \& Kallman, H. J. Encoding and use of detail information in picture recognition. Journal of Experimental Psychology: Human Learning and Memory, 1979, 5, 197-211.

MurPhy, B. J. Pattern thresholds for moving and stationary gratings. Vision Research, 1978, 18, 521-530.

Murphy, B. J., Haddad, G. M., \& Steinman, R. M. Simple forms and fluctuations in the line of sight: Implications for motor theories of form processing. Perception \& Psychophysics, $1974,16,557-563$.

Murphy, B. J., Kowlen, E., \& Steinman, R. M. Slow oculomotor control in the presence of moving backgrounds. Vision Research, 1975, 15, 1263-1268.

Rumelhart, D. A multicomponent theory of the perception of briefly exposed visual displays. Journal of Mathematical Psychology, 1970, 7, 191-218.

Schor, C., \& Hallmark, W. Slow control of eye position in strabismic amblyopia. Investigative Ophthalmology, 1978, 17, 577-581.

Skavenski, A. A., Hansen, R. M., Steinman, R. M., \& Winterson, B. J. Quality of retinal image stabilization during small natural and artificial body rotations in man. Vision Research, 1979, 17, 675-683.

Sperling, G. A model for visual memory tasks. Human Factors, $1963,5,19-31$.

Sperling, G. The description and luminous calibration of cathode ray oscilloscope visual displays. Behavior Research Methods \& Instrumentation, 1971, 3, 148-151.

Sperling, G., Budiansky, J., Spivak, J. G., \& Johnson, M. C. Extremely rapid usual search: The maximum rate of scanning letters for the presence of a numeral. Science, 1971, 174, 307-311.

Sperling, G., \& Melchner, M. J. Visual search, visual attention and the attention operating characteristic. In J. Requin (Ed.), Attention and performance VII. Hillsdale, N.J: Erlbaum, 1978.

Steinman, R. M., \& Collewisn, H. Binocular retinal image motion during active head rotation. Vision Research, in press.

Steinman, R. M., Cunitz, R. J., Timberlake, G. T., \& Herman, M. Voluntary control of microsaccades during maintained monocular fixation. Science, 1967, 155, 1577-1579.

Steinman, R. M., Haddad, G. M., Skavenski, A. A., \& Wyman, D. Miniature eye movement. Science, 1973, 181, 810819.

Winterson, B. J., \& Collewijn, H. Microsaccades during finely guided visuomotor tasks. Vision Research, 1976, 16, $1387-1390$.

Wolf, W., Hauske, G., \& LuPP, U. How presaccadic gratings modify postsaccadic modulation transfer function. Vision Research, 1978, 18, 1173-1179.

\section{NOTES}

1. Results similar to Sperling and Melchner's (1978) have been obtained by Haber and Nathanson (1969), who used a different task-memory for a sequence of single letters-and found the same performance for 10 -msec presentations followed by $150 \mathrm{msec}$ of darkness as they did for 150 -msec presentations followed by 10 msec of darkness.

2. It is well known that knowledgable subjects experienced in eye movement experiments, as well as naive, inexperienced sub- jects, can suppress saccades for many seconds after simple verbal instructions (Haddad \& Steinman, 1973; Steinman, Cunitz, Timberlake, \& Herman, 1967; Winterson \& Collewijn, 1976). Recently, the same results have been obtained for naive subjects with amblyopia, who can suppress saccades for many seconds after verbal instructions despite the presence of the high drift velocities characteristic of amblyopia (Ciuffreda, Kenyon, \& Stark, 1979; Schor \& Hallmark, 1978).

Subjects can also suppress saccades for many seconds with many different kinds of stimuli, for example, simple forms (Murphy, Haddad, \& Steinman, 1974), large flickering fields (Haddad \& Winterson, 1975), and moving backgrounds (Murphy, Kowler, \& Steinman, 1975), and while performing visual, cognitive, and visuomotor tasks, for example, making contrast threshold judgments about a moving sinusoidal grating (Murphy, 1978), counting visual items (Kowler \& Steinman, 1977, 1979), and threading a needle and aiming and shooting a rifle (Winterson \& Collewijn, 1976). [See also Kowler and Steinman (in press) and Steinman, Haddad, Skavenski, and Wyman (1973) for further reviews of this material.]

3. A separate $p$ was estimated for each pair of continuous presentations at $T$ and $2 \mathrm{~T} \mathrm{msec}$, rather than a single $\mathrm{p}$ for the entire curve. The separate ps were estimated so that the procedure used to test the model with continuous presentations would be comparable to the procedure used with two-flash presentations, in which performance with two flashes at $2 \mathrm{~T}$ msec and performance with a single flash at $T$ msec were used to estimate $p$.

4. An extreme case illustrates why predictions would be too large. If a subject scored $50 \%$ correct for a presentation time of $T$ msec and all targets had been of about the same difficulty, then the predicted performance for a presentation time of $2 \mathrm{~T}$ msec would be $75 \%$ correct (see Equation 1). But, suppose a subject scored $50 \%$ correct because half of the targets were very difficult and were correct $0 \%$ of the time, and half were very easy and were correct $100 \%$ of the time. Then, according to Equation 1, predicted performance for two independent presentations of each of these kinds of targets would still be $0 \%$ and $100 \%$ correct. Thus, overall performance in $2 \mathrm{~T} \mathrm{msec}$ would still be only $50 \%$ correct. Failure to separate targets into the easy and difficult categories would lead to a prediction of $75 \%$ correct, which would be too large.

5. The model treats differences between performance with displaced and nondisplaced presentations as due to statistical fluctuations. Nevertheless, the value of the estimated parameter $p$ for displaced presentations typically proved to be slightly smaller than values for the nondisplaced presentations (see values of $P_{1}$ in Table 1). The differences, however, were too small to approach statistical significance in the individual cases.

6. In the single-flash presentations, where we expect independent sampling to fail because the subject is not getting as much information in the second (dark) half of the exposure interval as in the first half, the model does fail significantly. Predictions based on performance with single-flash presentations at $T$ msec and at $2 \mathrm{~T}$ msec were almost always too high for performance at $2 \mathrm{~T} \mathrm{msec}$. The differences between observed and predicted performance were significant in three of four cases for subject E.K. (at $100 \mathrm{msec}, \chi^{2}=10.36$, df $=3, \mathrm{p}<.05$; at $200 \mathrm{msec}, \chi^{2}=$ 10.31, df $=4, \mathrm{p}<.05$; and at $400 \mathrm{msec}, \chi^{2}=10.34$, df $=4$, $\mathrm{p}<.05$ ) and in two of four cases for subject B.F. (at $400 \mathrm{msec}$, $\chi^{2}=9.34$, df $=3, p<.05 ;$ and at $800 \mathrm{msec}, \chi^{2}=19.58$, $\mathrm{df}=4, \mathrm{p}<.01)$.

(Received for publication July 23, 1979; revision accepted November 8,1979 .) 